\title{
Accuracy of a new drilling system (NDS) for paralleling implants: A pilot study
}

Auriol Muerza B' ${ }^{1}$, Revilla-Alonso MG ${ }^{1}$, Salomó-Coll O' ${ }^{1}$, Gargallo-Albiol J1

1 Universitat Internacional de Catalunya. International Master in Oral Surgery. Department of

Oral and Maxillofacial Surgery. Barcelona. Spain

\section{Abstract}

- Background: Surgical guides are used to assist dental implant placement in order to achieve an accurate position. The avoidance of these devices may lead to inaccuracies in the three dimensional optimal position, which may further result in undesirable functional and aesthetic results.

- Objective: To determine the accuracy of a new drilling system (NDS) evaluating the position and parallelism of implants placed with NDS, compared to a system which uses a radiological splint transformed into a surgical guide.

- Materials \& Methods: 10 posterior bilaterally edentulous patients requiring two adjacent teeth were included. A radiological splint was made and a CBCT was performed. The two drilling protocols were randomly assigned and the radiological stent was adapted to each drilling system. Periapical radiographs were taken.

- Results: The mean deviation for the mesial implant of the contro group was $-1^{\circ}$ (Standard Deviation $13,25^{\circ}$ ) and for the test group was $3,95^{\circ}$ (SD $2,34^{\circ}$ ). The deviation of the distal implant for the control group was $1,26^{\circ}\left(\operatorname{SD~} 10,45^{\circ}\right)$ and for the test group 8,76 (SD 1,13 $)$. No statistical differences were found between the two groups $(p>$ 0.05 ) regarding neither implants position nor implants divergence.

- Conclusions: The new drilling implant system (NDS) described is made to improve the positioning of implants during the surgery. Despite its limitations, this guide helps the surgeon placing the implants into the desired position, either parallel one to another or at preconceived angulations.

\section{Background and Aim}

The benefit of the computer-assisted implant planning and subsequent template-guided implant placement is a thorough preoperative diagnostic and a more predictable implantation procedure with respect to anatomical structures and prosthetic aspects.

Despite some disadvantages of the splints, such as possible instability or overheating of the bone, it is highly recommended to use them in order to know the final position of the restoration, and subsequently, the implant position.

The purpose of this study was to prove the accuracy of a new bur system to parallelize the implants compared with the standard protocol used in the clinic of the Universitat Internacional de Catalunya (UIC).

\section{Methods and Materials}

Patients enrolled were missing their posterior teeth in the maxilla or mandible, and needing at least two implants to be placed bilaterally with no posterior remaining teeth.

A diagnostic wax-up was done and a radiological splint was then performed. Afterwards, a preoperative computed tomography was performed.

After the randomized assignment of the groups, the surgical stent was adapted to the drilling system. The pilot drill was used followed by the conventional drilling protocol recommended by the manufacturer.

Periapical $x$-rays were taken after the surgery as well as intraoral and cast model photographs. 3 months after implant placement, periapical $x$ rays and impressions were taken.

\section{Results}

The evaluation of the mesio-distal position and the divergence of the implants was assessed through the $x$-rays performed after the surgery. The evaluation of the bucco-lingual position of the implants was not assessed because not all the cases underwent the time required for the osseointegration, so direct impressions to the implants could not been performed.

Mean and Standard Deviations were calculated, setting the adjacent teeth as a reference $(0)^{\circ}$ for angles assessment. Distal implant inclination was also calculated with the reference tooth.

\begin{tabular}{|c|c|c|c|c|c|}
\hline GROUP & Sites & Gender & Mesial Implant & Distal Implant & Divergence \\
\hline \multirow{5}{*}{ CONTROL } & $\# 1$ & $\mathrm{M}$ & $0^{\circ}$ & $-1,6^{\circ}$ & $-1,6^{\circ}$ \\
\cline { 2 - 6 } & $\# 2$ & $\mathrm{H}$ & $8^{\circ}$ & $6,9^{\circ}$ & $1,1^{\circ}$ \\
\cline { 2 - 6 } & $\# 4$ & $\mathrm{M}$ & $6^{\circ}$ & $13^{\circ}$ & $-7^{\circ}$ \\
\cline { 2 - 6 } & $\# 7$ & $\mathrm{M}$ & $-19^{\circ}$ & $-6^{\circ}$ & $-13^{\circ}$ \\
\cline { 2 - 6 } & $\# 8$ & $\mathrm{M}$ & $0^{\circ}$ & $-6^{\circ}$ & $-6^{\circ}$ \\
\hline \multirow{5}{*}{ TEST } & $\# 3$ & $\mathrm{H}$ & $2^{\circ}$ & $9,9^{\circ}$ & $-7,9^{\circ}$ \\
\cline { 2 - 6 } & $\# 5$ & $\mathrm{M}$ & $4,3^{\circ}$ & $9,6^{\circ}$ & $-5,3^{\circ}$ \\
\cline { 2 - 6 } & $\# 6$ & $\mathrm{M}$ & $3,2^{\circ}$ & $8,1^{\circ}$ & $-4,9^{\circ}$ \\
\cline { 2 - 6 } & $\# 9$ & $\mathrm{M}$ & $7^{\circ}$ & $8^{\circ}$ & $-1^{\circ}$ \\
\cline { 2 - 6 } & $\# 10$ & $\mathrm{M}$ & $3,3^{\circ}$ & $8,2^{\circ}$ & $-4,9^{\circ}$ \\
\hline
\end{tabular}

Table 1. Description of the values of the control and test group.

\begin{tabular}{|c|c|c|c|}
\hline GROUP & IMPLANT & MEAN & SD \\
\hline \multirow{2}{*}{ CONTROL } & MESIAL & -1 & 13,25 \\
\cline { 2 - 4 } & DISTAL & 1,26 & 10,45 \\
\hline \multirow{2}{*}{ TEST } & MESIAL & 3,95 & 2,34 \\
\cline { 2 - 4 } & DISTAL & 8,76 & 1,13 \\
\hline
\end{tabular}

Table 2. Means and Standard Deviations for test and control implant groups.

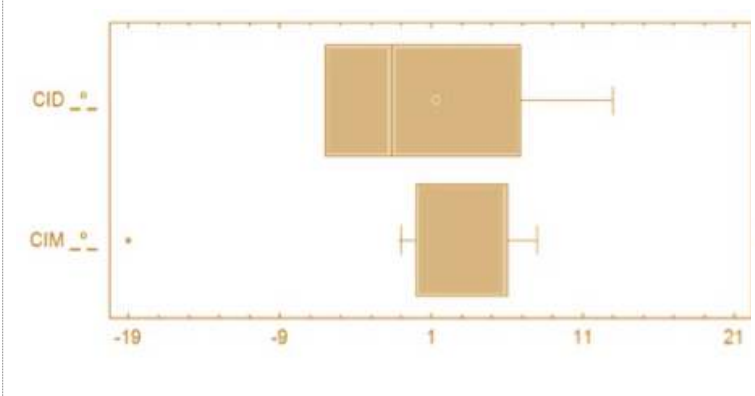

Fig 1. Box plot comparing Mesial vs. Distal implants (Control)
int comparing Mesias

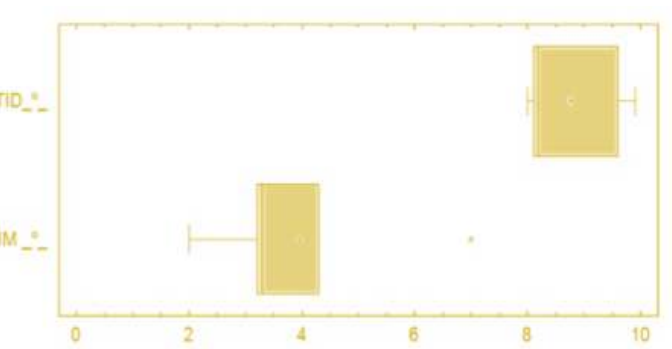

Fig 2. Box plot comparing Medial vs. Distal mplants (Test)

\section{Conclusions}

Both drilling systems proved a valid option to parallelize implants, although NDS presented some limitations. The use of well-designed surgical splints, which guide us on the final restoration, is essential to get a good parallelism and a correct position of the implants.

However, the surgical stent should always be supported by intraoperative assessment and clinical experience of the surgeon.

\section{References}

Jee-Ho L., Ji-Man P., Soung-Min K. (2013) An assessment of template-guided implant
surgery in terms of accuracy and related factors. Journal of Advanced Prosthodontics surgery in tem
$5(4): 440-47$

2. Valente F., Schiroli G., Sbrenna A. (2009) Accuracy of computer-aided oral implant surgery: a clinical and radiographic study. International Journal of Oral \& Maxillofacial
Implants 24(2): 234-42

3. Scheider D., Marquardt P., Zwahlen M., Jung RE. (2009) A systematic review on the accuracy and the clinical outcome of comput
Clinical Oral Implants Research 20(4): 73-86

4. Van Assche N., Van Steenberghe D., Guerrero M. E., Hirsch E. (2007) Accuracy of implant placement based on pre-surgical planning of three-dimensional cone-beam
images: a pilot study. Journal of Clinical Periodontology. 34(9): 816-21

5. Fortin T., Champleboux G., Lormée J., Coudert JL. (2000) Precise dental implant placement in bone using surgical guides in conjunction with medical imaging techniques.
Journal of Oral Implantology $26(4): 300-3$ 6. Widmann G., Bale RJ. (2006) Accuracy in computer-aided implant surgery: a review.
International' Journal of Oral \& Maxillofacial Implants 21(2): 305-13 